\title{
Resveratrol Protects Against Pulmonary Arterial Hypertension in Rats via Activation of Silent Information Regulator 1
}

\author{
Lei Yu ${ }^{a}$ Yingfeng Tu ${ }^{b} \quad$ Xueling Jia ${ }^{a}$ Kun Fanga Li Liu $^{c}$ Lin Wand Chuanying Xianga \\ Yanan Wang ${ }^{a}$ Xiangju Sun ${ }^{b}$ Tianyou Liu ${ }^{e}$ Dejun Yu $^{f}$ Weiwei Cao ${ }^{a}$ Yinli Songa \\ Yuhua Fan ${ }^{a}$ \\ ${ }^{a}$ College of Pharmacy, Harbin Medical University-Daqing, Daqing, ${ }^{\mathrm{b}}$ Department of Cardiology, the \\ Fourth Hospital of Harbin Medical University, Harbin, 'Department of Anesthesiology, the Third \\ Hospital of Harbin Medical University, Harbin, ${ }^{d}$ Department of Breast Surgery, the Third Hospital of \\ Harbin Medical University, Harbin, e Department of Colorectal Surgery, the First Affiliated Hospital of \\ Harbin Medical University, Harbin, fDepartment of Laboratory Diagnosis, the fifth affiliated hospital of \\ Harbin Medical University, Daqing, China
}

\section{Key Words}

Resveratrol (Rev) • Pulmonary arterial hypertension (PAH) • Silent information Regulator 1 (SIRT1) • Mitochondrial

\begin{abstract}
Background/Objectives: The polyphenol resveratrol (Rev) has been found to exhibit various beneficial effects including prevention of pulmonary arterial hypertension (PAH). The present study was designed to investigate the action and potential mechanism of Rev on PAH, focusing on the role of SIRT1 (Silent Information Regulator 1 ) in apoptosis of pulmonary artery smooth muscle cells (PASMCs). Methods: PAH rats were established by exposure to hypoxia for 21 days. Rev and SRT1720 (a selective SIRT1 activator) were used to reverse PAH by gavaging rats. PASMCs were confronted with hypoxia for $24 \mathrm{~h}$ or $48 \mathrm{~h}$ and were then treated with Rev or SRT1720 in vitro. Western blot was performed to detect the protein expression of SIRT1. CCK-8 and scratch wound experiments were carried out to verify cell proliferation. In addition, the TUNEL positive assay and flow cytometry assay were used to measure PASMC apoptosis. Mitochondrial permeability transition (mPT) was identified by confocal microscopy. Right ventricular systolic pressure (RVSP) was determined with a Gould pressure transducer, and right ventricular hypertrophy $(\mathrm{RVH})$ was determined by weighing the cardiac muscle. Results: We demonstrated that Rev could reverse the remodelling of the pulmonary vasculature, thus contributing to alleviating the severity of PAH. Down-regulation of SIRT1 was observed in PAH, but administration of Rev had no obvious effect on the protein expression of SIRT1. In addition, Rev could induce mitochondrial swelling and nuclear pyknosis, leading to small, dense, and dysmorphic mitochondria in rats exposed to hypoxia alone. Rev treatment inhibited

L. Yu and Y. Tu contributed equally to this work.


PASMC proliferation in a dose-dependent manner in vitro. Incubation with SRT1720, a specific activator of SIRT1, significantly retarded PASMC proliferation and promoted PASMC apoptosis in vitro. The mechanism could be associated with inducing MPT damage in PASMCs. Rev and SRT1720 treatment mitigated RVSP and reduced RVH. Conclusion: Rev produced a beneficial effect partially by enhancing the activation of SIRT1, thus improving RVSP and reducing RVH. SIRT1 activation increased PASMC apoptosis by inducing $\mathrm{MPT}$ dysfunction, which might be a novel future strategy for the treatment of PAH.

\section{Introduction}

Chronic hypoxia-induced pulmonary arterial hypertension (PAH) is a life-threatening disease that is characterized by progressive remodelling of the pulmonary vasculature, which can ultimately lead to right heart failure and death $[1,2]$. The hallmark of terminal $\mathrm{PAH}$ is the development of plexiform lesions resulting from the excessive proliferation and migration of pulmonary artery endothelial cells (PAECs) and pulmonary artery smooth muscle cells (PASMCs) [3, 4]. Several traditional methods, including administration of calcium-channel blockers and anticoagulants, have limited functions. Currently, the therapies for PAH such as endothelin receptor antagonists $[5,6]$, phosphodiesterase type 5 inhibitors [7], and prostacyclin analogues [8] are considered primary treatments. Although these therapies could partially improve the quality of life in patients with $\mathrm{PAH}$, congenital heart disease (PAH-CHD) carries a poor prognosis [9]. Therefore, novel therapeutic strategies and newer agents are needed to target multiple pathways involved in vasoconstriction, cellular proliferation, the inflammatory response, and so on [10]. For instance, in experimental therapies, KCNK3 inhibition enhanced cell proliferation, vasoconstriction, and inflammation, but in vivo pharmacological activation of KCNK3 alleviated monocrotaline-induced PH [11]. In addition, herbs have shown extensive beneficial effects, such as puerarin, which promotes the apoptosis of PASMCs in hypoxia by acting on mitochondria-dependent pathways [12].

Currently, multiple antioxidants and small molecules have also been identified as having beneficial effects against the deleterious effects of PAH in different experimental models [1316]. Gene manipulation research has also confirmed the effects of antioxidants, which can be applied in prevention strategies against PAH. Resveratrol (Rev, 3,5,4-trihydroxystilbene), a polyphenol isolated from grapes and polygonum cuspidatum, has been shown to possess antioxidative properties [17]. A recent study showed that Rev could reverse monocrotalineinduced pulmonary vascular remodelling and prevent the progression of PAH $[18,19]$. However, the exact anti-PAH function of Rev has not been fully uncovered until now.

Here, we investigated the anti-PAH function of Rev in vivo and in vitro, and we determined that Rev reversed the remodelling of the pulmonary vasculature and decreased the number of mitochondria in PASMCs. We also showed that SRT1720, a specific activator of SIRT1, decreased cell viability and induced PASMC apoptosis by disrupting the action of mitochondria in this study.

\section{Materials and Methods}

\section{Animals}

Healthy male Wistar rats (200-300 g) were purchased from the Experimental Animal Center of Harbin Medical University (Grade II), China. The rats were maintained under standard animal room conditions (temperature $23 \pm 1^{\circ} \mathrm{C}$; humidity $55-60 \%$ ). Food and water were freely available throughout the experiment. All procedures for the use of animals were approved by the Institutional Animal Care and Use Committee of Harbin Medical University [Protocol (2009)-11]. The use of animals was compliant with the Guide for the Care and Use of Laboratory Animals published by the US National Institutes of Health (NIH Publication No. 85-23, revised 1996). 


\section{Cellular Physiology Cell Physiol Biochem 2017;42:55-67 and Biochemistry Published onIme: IVlay L2, $2017 \quad$\begin{tabular}{l|l} 
DOI: 10.1159/000477115 2017 The Author(s). Published by S. Karger AG, Basel \\
www.karger.com/cpb
\end{tabular}}

Yu et al.: Resveratrol Prevents PAH through SIRT1 Activation

Induction of HPVR (hypoxic pulmonary vascular remodelling)

The rat models were randomly assigned to 21 days of normal conditions, hypoxia-induced pulmonary vascular remodelling (HPVR) conditions, or Rev $(25 \mathrm{mg} / \mathrm{kg} / \mathrm{day}$, Sigma Chemical Co., St. Louis, MO, USA) plus hypoxia conditions [20]. Briefly, hypoxia-induced HPVR involved a fractional inspired oxygen of 0.21 and 0.12 , as described previously [21]. At the end of the 21-day exposure period, we anaesthetized each rat and quickly removed the lungs.

\section{Cell isolation and cultivation}

The intrapulmonary arteries were carefully de-endothelialized and then digested with enzymatic solution: $0.15 \%$ type II collagenase (Worthington) and $0.15 \%$ BSA in PBS for $1 \mathrm{~h}$ at $37^{\circ} \mathrm{C}$. The digested pulmonary arterial smooth muscle cells (PASMCs) were then kept in DMEM supplemented with $10 \%(\mathrm{v} / \mathrm{v}$ ) FBS (foetal bovine serum), 1\% (w/v) streptomycin and 1\% (w/v) penicillin for 3-5 days in a humidified incubator with $5 \% \mathrm{CO}_{2}$ at $37^{\circ} \mathrm{C}$. PASMCs at passages 2-4 were used for further experiments. Before each experiment, the cells were incubated in serum-free low-glucose DMEM for $24 \mathrm{~h}$ to stop growth. For hypoxic cultivation, the cells were grown in a Tri-Gas incubator (HF100; Heal Force) providing an atmosphere of $92 \% \mathrm{~N}_{2} / 5 \% \mathrm{CO}_{2} / 3 \% \mathrm{O}_{2}$ as described previously [22].

\section{Cell viability assay}

The cell viability was calculated using the Cell Counting Kit-8 (CCK-8) (Beyotime Biotechnology, Jiangsu, China) according to the manufacturer's protocol. Briefly, the pulmonary arterial smooth muscle cells (PASMCs) were seeded on 96-well plates in DMEM with 5\% FBS for $24 \mathrm{~h}$ in normoxia. Then, the cells were treated at $80 \%$ confluence using SRT 1720 for $24 \mathrm{~h}$ or $48 \mathrm{~h}$. Finally, the viable cells were detected using the Cell Counting Kit-8, where the absorbance for each sample was assessed at $450 \mathrm{~nm}$ using a microplate reader (TECAN, Salzburg, Austria). In addition, a calcein-AM/PI dual-staining assay (Invitrogen-Molecular Probes, Carlsbad, CA) was performed to further document the effect of SRT1720 on cell apoptosis. The calcein-AM/PI assay relies on the intracellular esterase activity within living cells. Living cells are stained with green fluorescence, and dead cells are stained red by PI. PASMCs were treated with SRT1720 for 24 $\mathrm{h}$ in DMEM with 5\% FBS under hypoxic conditions. Fluorescence was analysed by microscopy (Olympus, Tokyo, Japan).

\section{TUNEL assay}

DNA fragmentation in PASMCs was measured using the TUNEL assay (Cell Death Detection Kit, Roche Biochemicals, Mannheim, Germany). Briefly, air-dried slides were fixed with $4 \%$ paraformaldehyde for 30 min at room temperature, cleaned three times with PBS for $10 \mathrm{~min}$, and then permeabilized with $1 \%$ Triton $\mathrm{X}-100$ for $4 \mathrm{~min}$ at $4^{\circ} \mathrm{C}$. Then, the TdT-labelled nucleotide mix was added to each slide and incubated at $37^{\circ} \mathrm{C}$ for $60 \mathrm{~min}$ in a dark environment. The slides were washed twice with PBS and then counterstained with 10 $\mathrm{mg} / \mathrm{ml} \mathrm{4,6-diamidino-2-phenylindole} \mathrm{(DAPI)} \mathrm{for} 5 \mathrm{~min}$ at $37^{\circ} \mathrm{C}[23]$.

\section{Quantitative Reverse-Transcription Polymerase Chain Reaction (qRT-PCR)}

Total RNA samples from cultured PASMCs were isolated using Trizol reagent (Invitrogen, Carlsbad, CA) according to the manufacturer's protocols. Total RNA $(1 \mu \mathrm{g})$ was then reverse transcribed using the High-Capacity cDNA Reverse Transcription Kit (ToYoBo) to obtain cDNA. The SYBR Green PCR Master Mix Kit (ToYoBo) was used in the quantitative reverse-transcription polymerase chain reaction (qRT-PCR) to quantify RNA levels of SIRT1 (Silent Information Regulator 1). GAPDH was used as an internal control. qRTPCR was performed on the 7300 FAST Real-Time PCR System (Applied Biosystems) for 40 cycles. Rat SIRT1: (forward) 5'-GGCACATGCCA GAGTCCAAG-3' and (reverse) 5'-CACTCCGGTCTGTCAGCATCA- 3'; and GAPDH: (forward) 5'-GCTGGGGCTCACCTGAAGG-3' and (reverse) 5'-GGAT GACCTTGCCCACAGCC-3'.

\section{Scratch-Wound assay}

Confluent PASMCs cultured in 6-well plates were scratched with pipette tips, which led to a 1-mmwide lane per well, and the ablated cells were cleaned with PBS. Then, the cells were treated with vehicle or chemicals of interest with 5\% FBS DMEM. Wounded areas were photographed at time point zero. After the 24-h treatment, photographs were taken of the same recorded areas. 
Western blot Analysis

PASMCs were treated with Rev in DMEM with 5\% FBS for 24 h in hypoxic conditions. Proteins were solubilized and extracted with $200 \mu \mathrm{l}$ of lysis buffer and incubated for $30 \mathrm{~min}$ on ice. Next, the lysates were sonicated and centrifuged at 12,000 g for $15 \mathrm{~min}$ and the insoluble fractions were discarded. Equal amounts of protein $(\sim 60 \mu \mathrm{g})$ from each sample were subjected to electrophoresis on an SDS-polyacrylamide gel and transferred onto nitrocellulose membranes (Millipore, USA). After $1 \mathrm{~h}$ of incubation with 5\% non-fat dry milk powder, the membranes were probed with primary antibody to SIRT1 at 1:4000 for $16 \mathrm{~h}$ at $4{ }^{\circ} \mathrm{C}$. Blots were then incubated with horseradish peroxidase-conjugated secondary antibodies and enhanced chemiluminescence reagents.

\section{Measurements of right ventricular systolic pressure}

Male Wistar rats were randomly assigned to one of four groups, including normoxia (Nor), hypoxia (Hyp), hypoxia+Rev (Hyp+Rev), and hypoxia+SRT1720 (Hyp+SRT) treatment groups. In the Hyp+SRT group, rats were co-treated with SRT1720 (25 mg/kg/day) for 21 days, which was purchased from selleck.cn (Shanghai, China). After 21 days of hypoxic treatment, rats were anesthetized with $2 \%$ sodium pentobarbital powder by intraperitoneal injection ( $40 \mathrm{mg} / \mathrm{kg}$ ), and then pressure measurements were performed. Right ventricular systolic pressure (RVSP) was detected using a single lumen catheter $(0.012$ " $\times 0.016$ " silicone tubing) through the right external jugular vein. After placement of the catheter into the right ventricle, RVSP was measured with a Gould pressure transducer positioned at the mid-thorax of the animal and a Gould multichannel recorder. Proper catheter location was verified by the waveform of the pressure tracing.

\section{Evaluation of right ventricular hypertrophy}

After removal of the atria, the hearts were dissected and then the right ventricle (RV) was separated from the left ventricle and septum $(\mathrm{LV}+\mathrm{S})$ under a dissecting microscope. The dissected wet samples were weighed after drying at $90^{\circ} \mathrm{C}$ for $40 \mathrm{~h}$ to obtain the ratio of $\mathrm{RV} / \mathrm{LV}+\mathrm{S}$. The ratio of $[\mathrm{RV} /(\mathrm{LV}+\mathrm{S})]$ was measured as the right ventricular hypertrophy index (RVHI) as described by Abe et al. [24].

\section{Statistical analysis}

All quantitative data are expressed as the mean \pm SEM. Statistical analysis was performed with Student's t-test or one-way ANOVA followed by Dunnett's test where appropriate. $p<0.05$ was considered statistically significant [25].

\section{Results}

Resveratrol (Rev) reverses pulmonary vascular remodelling and contributes to mitochondrial dysfunction

The morphology of the pulmonary vessels was measured with Hematoxylin-Eosin (HE) staining. The results showed that the pulmonary vascular walls in HPVR rats were thicker than the vessels from normal rats as observed with HE staining. More importantly, we found that the increase was substantially reversed by administration of Rev, a non-specific activator of SIRT1 (Silent Information Regulator 1), in vivo (Fig. 1B). These representative images are shown in Fig. 1A. To determine the mechanism of Rev intervention on pulmonary vascular remodelling, the alteration of mitochondria was measured using electron microscopy (EM). EM results indicated that the mitochondria and the nuclei were intact in the PASMCs of normal rats. Simultaneously, we found that the mitochondria were small, dense, and dysmorphic. Also, the number of mitochondria was increased in the PASMCs of HPVR rats. In contrast, after administration of Rev, the increase in the number of mitochondria was reduced, most of the mitochondria were swollen, and nuclear pyknosis was evident (Fig. 1C). To determine the role of Rev in hypoxia-induced cell proliferation, cell viability was measured using a CCK8 assay and Trypan blue dye exclusion. The CCK- 8 assay data indicated that $\operatorname{Rev}(0,5,15,30$, $50 \mu \mathrm{M}$ ) could reduce the viability of PASMCs that were exposed to hypoxia for $24 \mathrm{~h}$ in a dosedependent manner (Fig. 1D). In addition, Trypan blue dye exclusion showed that the viability of the PASMCs was also remarkably attenuated by treatment with $\operatorname{Rev}(30,50 \mu \mathrm{M})$ following 
Fig. 1. Effects of Resveratrol (Rev) on pulmonary vascular remodelling, mitochondrial morphology and cell proliferation. (A) Representative images of $\mathrm{H} \& \mathrm{E}$ staining are shown. (B) H\&E staining indicated that the wall thickness was predominantly decreased by treatment with Rev in HPVR. (C) Electron microscopy images of the pulmonary vasculature in rats. White arrowheads indicate mitochondria. $\mathrm{N}$ represents nuclei. (D) PASMCs were exposed to different concentrations of Rev for $24 \mathrm{~h}$ in hypoxia. Viable cells were measured by the CCK-8 assay. (E) The trypan blue exclusion assay showed that high doses of Rev could decrease hypoxiamediated PASMC proliferation. Data are expressed as the mean \pm SEM. ${ }^{*} p<0.05$ or ${ }^{* *} p<0.01$ vs. Nor group; $\# p<0.05$ vs. Hyp-treated group.

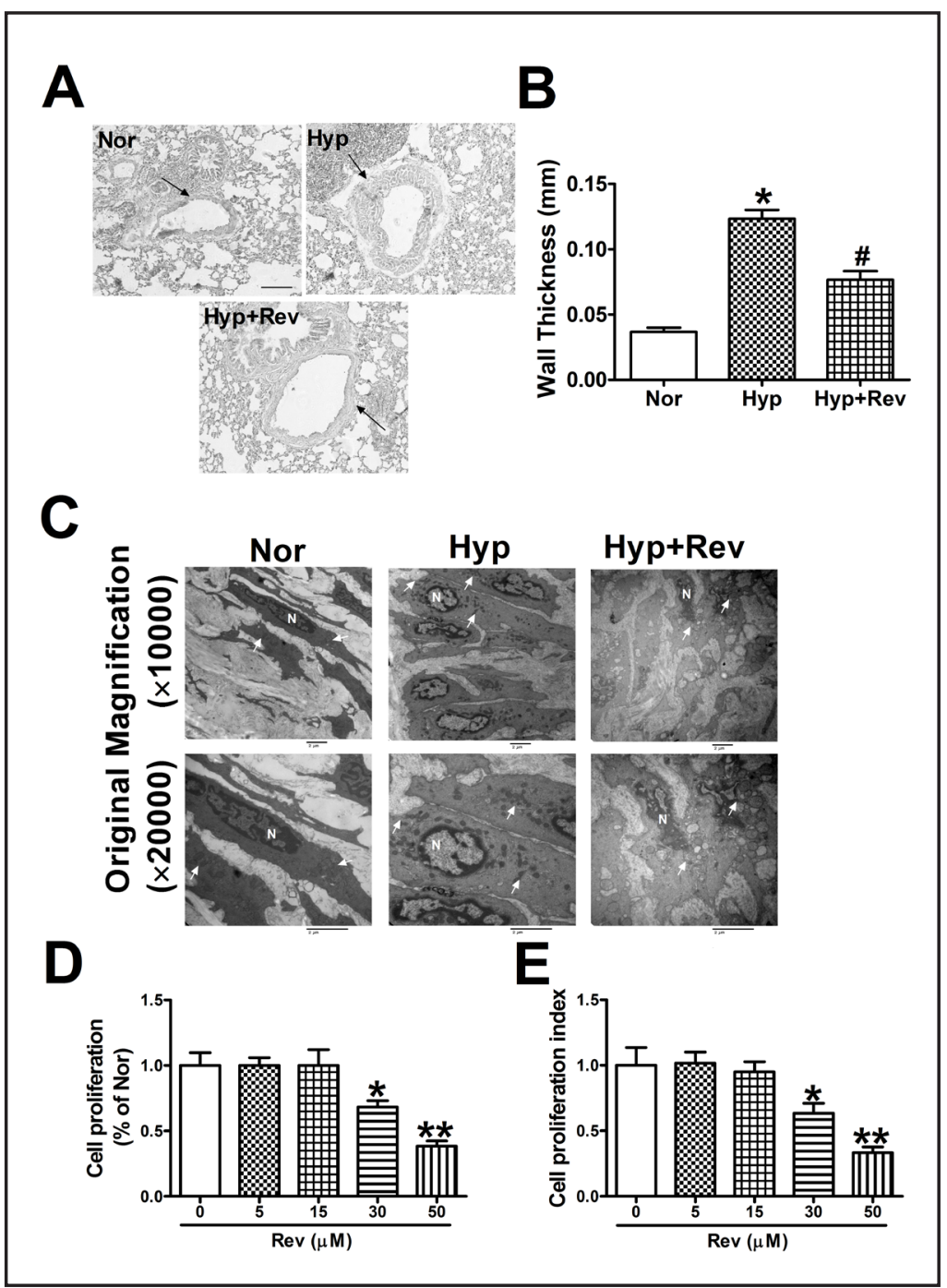

a 24-h exposure to hypoxia (Fig. 1E). This indicated that Rev reversal of pulmonary vascular remodelling was, at least in part, attributable to a reduction in the number of mitochondria and inhibition of cell proliferation.

Rev could not reverse the downregulation of SIRT1 induced by hypoxia

To determine the mechanism linking Rev with altered pulmonary vascular remodelling, western blot analysis was performed. As previously mentioned, Rev is a type of nonspecific activator of SIRT1. Therefore, we explored whether SIRT1 was involved in the progression of pulmonary vascular remodelling induced by hypoxia. PASMCs were incubated with Rev for $24 \mathrm{~h}$. We observed that the protein expression of SIRT1 was obviously reduced in rats exposed to hypoxia, but Rev could not reverse the protein expression of SIRT1 induced by hypoxia (Fig. 2A). Similarly, the data showed that the protein expression of SIRT1 was substantially reduced in PASMCs exposed to hypoxia alone and that Rev was unable to reverse the protein level of SIRT1 induced by hypoxia (Fig. 2B). In addition, the qRT-PCR results showed that the mRNA level of SIRT1 was also clearly decreased in rats and PASMCs after exposure to hypoxia. However, Rev could not elevate the downregulated mRNA level of SIRT1 mediated by hypoxia (Fig. 2C and 2D). These results indicated that the SIRT1 pathway might be involved in the process of hypoxia-induced pulmonary vascular remodelling, but the beneficial effect of Rev could not alter the protein level of SIRT1. 
Fig. 2. Effect of Rev on the level of SIRT1 mediated by hypoxia in rats and PASMCs. (A \& B) The protein expression of SIRT1 was reduced in rats and PASMCs exposed to hypoxia, but Rev had no such effect. (C \& D) mRNA levels of SIRT1 were obviously decreased in rats and PASMCs exposed to hypoxia. However, the mRNA level of SIRT1 was not altered by treatment with Rev. Data are expressed as the mean \pm SEM. ${ }^{*} p<0.05$ or $* * p<0.01$ vs. Nor group.
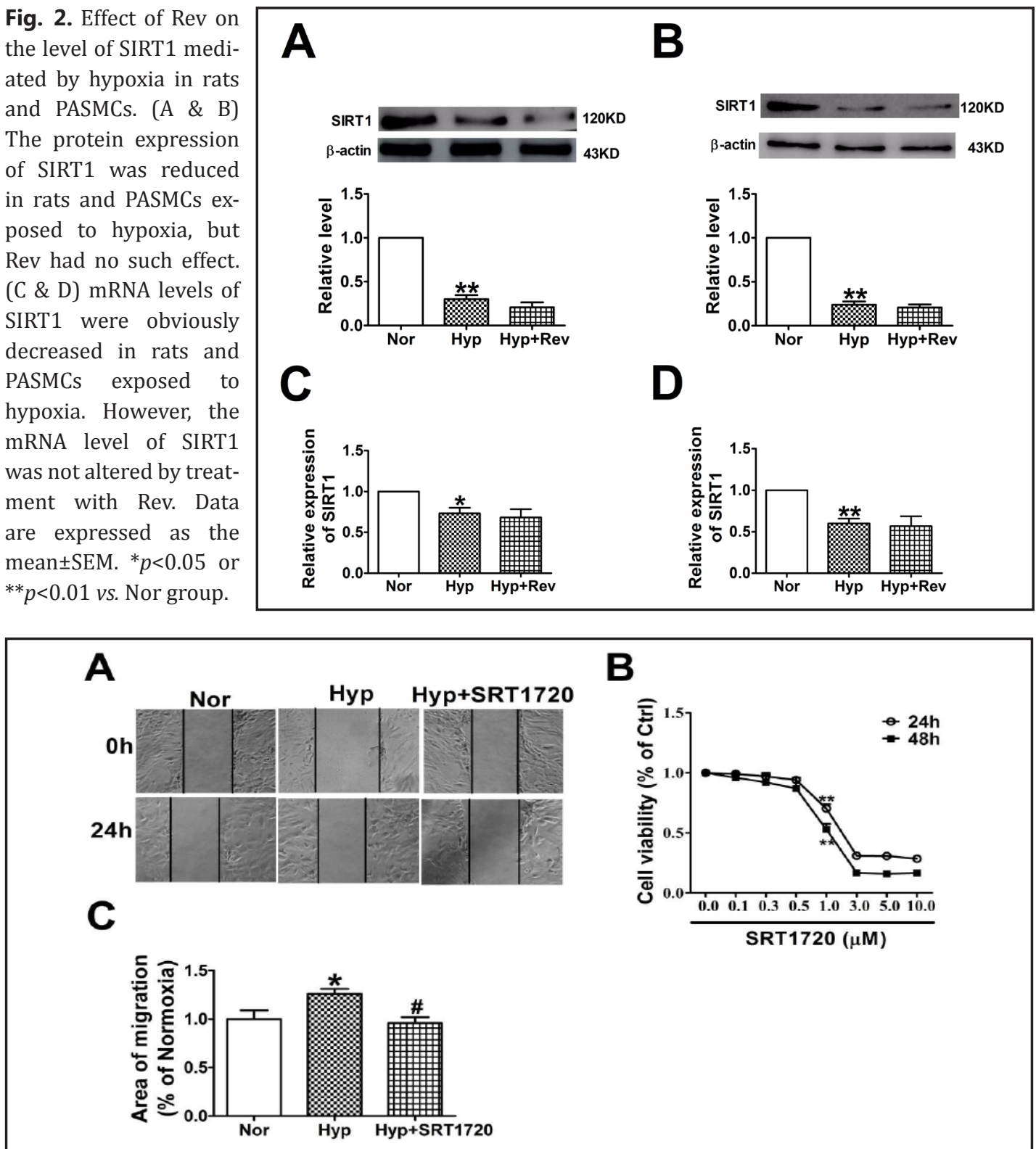

Fig. 3. SRT1720 attenuates PASMC proliferation by enhancing the activation of SIRT1. (A and C) The ScratchWound assay indicated that the proliferation of PASMCs was predominantly reduced by incubation with SRT1720 for $24 \mathrm{~h}$ under hypoxia. (B) Cell proliferation was inhibited in a dose-dependent manner by treatment with SRT1720 for $24 \mathrm{~h}$ or $48 \mathrm{~h}$ in hypoxia. Data are expressed as the mean \pm SEM. ${ }^{*} p<0.05$ or ${ }^{* *} p<0.01$ vs. Nor group; \#p<0.05 vs. Hyp group.

\section{Activation of SIRT1 by SRT1720 retards PASMC proliferation}

Based on the above results, SRT1720, which is known to be a specific activator of SIRT1, was used to generate the effect of SIRT1 activation on cell proliferation. To verify that Rev reversed pulmonary vascular remodelling through the SIRT1 pathway, the scratch-wound experiment and CCK-8 assay were performed. First, the effect of SIRT1 on PASMC proliferation was assessed using the CCK-8 assay. Treatment of the PASMCs with various concentrations of SRT1720 $(1,3,5$, and $10 \mu \mathrm{M})$ produced a marked decrease in cell viability after exposure to hypoxia at the 24-h and 48-h time points. We found that the dose $(3,5$, and $10 \mu \mathrm{M})$ of $\operatorname{Rev}$ caused greater than $70 \%$ inhibition in cell proliferation at the 24 -h and 48 -h time points (Fig. 3B) and that almost all PASMCs experienced death (not shown). Therefore, the 24-h 
Fig. 4. Incubation with SRT1720 promotes PASMC apoptosis. (A) Photographs of TUNEL-positive PASMCs. (B) Quantitative analysis of apoptotic nuclei. (C) Representative images of PASMCs treated with hypoxia or SRT1720. (D and E) Histogram of the cell populations of viable, early apoptosis (EAO), late apoptosis (LAO) and secondary necrosis (SNO) PASMCs. Data are expressed as the mean \pm SEM. ${ }^{*} p<0.05$ or ${ }^{* *} p<0.01$ vs. Nor group; $\# p<0.05$ or \#\#p<0.01 vs. Hyp-treated group.

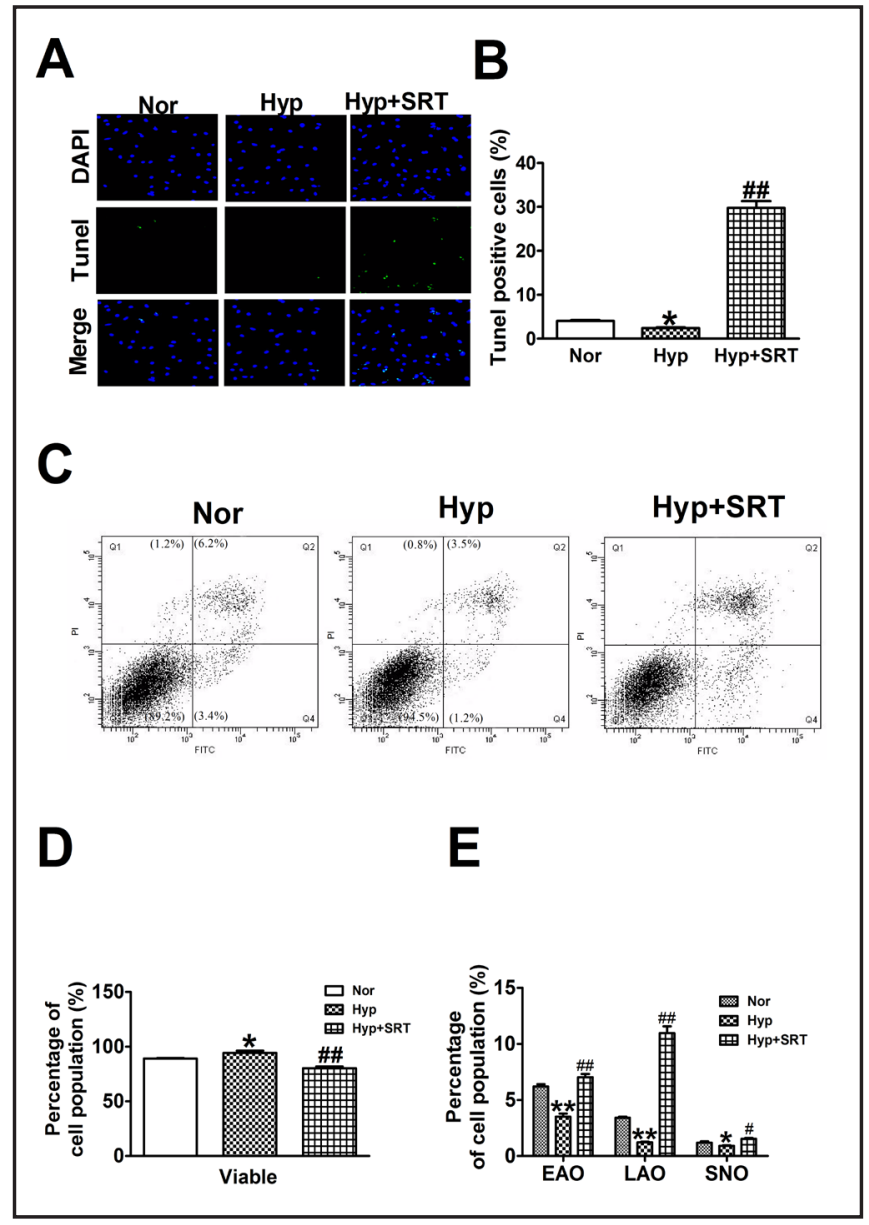

time point and a dose of SRT1720 or $1 \mu \mathrm{M}$ were selected for the following experiments. In addition, a scratch-wound assay was performed to verify the anti-proliferation effect of SRT1720. The results indicated that PASMCs incubated with SRT1720 showed substantial attenuation of hypoxia-mediated cell migration (Fig. 3C). Representative images are shown in Fig. 3A.

\section{SRT1720 treatment contributes to PASMC apoptosis}

The balance between cell proliferation and apoptosis is essential to the maintenance of homeostasis in multicellular organisms. To confirm whether activation of SIRT1 contributed to PASMC apoptosis, a TUNEL assay was performed. As shown in Fig. 4B, TUNEL-positive cells markedly decreased in PASMCs exposed to hypoxia compared with the normoxia group, but administration of SRT1720 (1 $\mu \mathrm{M})$ substantially elevated PASMC apoptosis compared with hypoxia treatment alone at the 24-h time point. Representative images are shown in Fig. 4A. To further clarify the effect of SRT1720 on apoptosis in PASMCs, a flow cytometry assay was also carried out. As shown in Fig. 4D and 4E, the number of apoptotic PASMCs was remarkably reduced during exposure to hypoxia for $24 \mathrm{~h}$, but administration with SRT1720 clearly promoted cell apoptosis. Representative images are shown in Fig. 4C. Altogether, the results from this study indicated that specific SIRT1 activation contributed to the amelioration of PAH.

SRT1720 incubation-induced apoptosis is associated with late mitochondrial permeability transition ( $m P T)$

Mitochondrial membrane permeabilization, including $\mathrm{mPT}$ and depolarization of $\Delta \Psi \mathrm{m}$, plays a key role in the early stages of cell apoptosis [26]. Previous studies indicated that 
Fig. 5. SRT1720 incubation increases cell apoptosis by inducing dysfunction of mitochondrial permeability transition (mPT). Calcein AMstained cells (green) are alive, and PI-stained cells (red) are dead. (A) Calcein fluorescence was obviously increased under hypoxia, but SRT1720 reduced calcein fluorescence. (B) PASMCs were also loaded with calcein $\mathrm{AM} / \mathrm{CoCl}_{2}$. (C) SRT1720 incubation could obviously decrease the intensity of mean calcein fluorescence in PASMCs under hypoxia. (D) Representative images showing apoptotic PASMCs treated with SRT1720. (E) The apoptotic cell rate was significantly higher in the SRT1720-treated group. Data are expressed as the mean \pm SEM. ${ }^{* *} p<0.01$ vs. Nor group; \#\#p<0.01 vs. Hyptreated group.

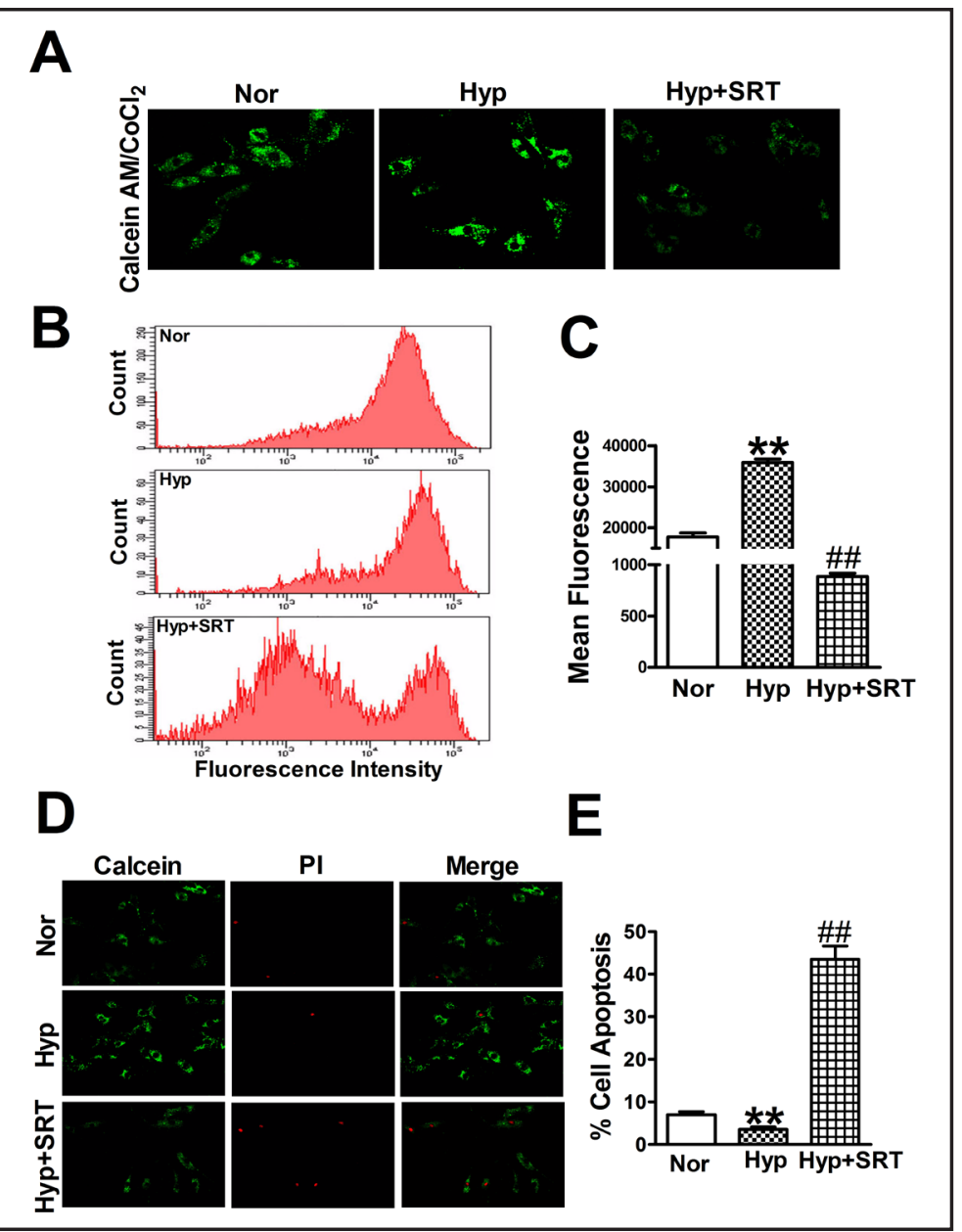

sustained opening of the mPT pore resulted in cell apoptosis. To assess whether SRT1720 promotes cell apoptosis by altering mPT, PASMCs were loaded with calcein AM (green, live cells)/ $\mathrm{CoCl}_{2}$. The data from the study showed that calcein fluorescence was obviously reduced in PASMCs incubated with SRT1720 for $24 \mathrm{~h}$ compared to those that were hypoxiatreated alone, indicating that $\mathrm{mPT}$ occurred and that $\mathrm{CoCl}_{2}$ entered the mitochondria to decrease calcein. The representative images are shown in Fig. 5A. Moreover, flow cytometry was performed to further document the effect of SRT1720 on mPT. As shown in Fig. 5C, the mean fluorescence was obviously attenuated by treatment with SRT1720. Representative images are shown in Fig. 5B. In addition, we further monitored the effect of SRT1720 on PASMC apoptosis at the 24-h time point. As expected, we found that SRT1720 caused an increase in DNA fragmentation in hypoxia. Confocal fluorescence images of calcein AM and PI (red, dead cells) co-stained cells are shown in Fig. 5D. The number of apoptotic cells was substantially elevated in the SRT1720-treated group (Fig. 5E). These data suggest that SIRT1 activation could impede $\mathrm{mPT}$, thus promoting cell apoptosis. So, it is reasonable to speculate that SIRT1 might be a potential therapeutic target to reverse pulmonary vascular remodelling and retard the progression of PAH.

Rev and SRT1720 improve right ventricular systolic pressure (RVSP) and mitigate right ventricular hypertrophy (RVH)

To further verify the beneficial effects of Rev and SRT1720, we measured the values of RVSP and RVH in the different treatment groups. The results indicated that both Rev and SRT1720 could decrease the level of RVSP induced by hypoxia (Fig. 6A). In addition, 


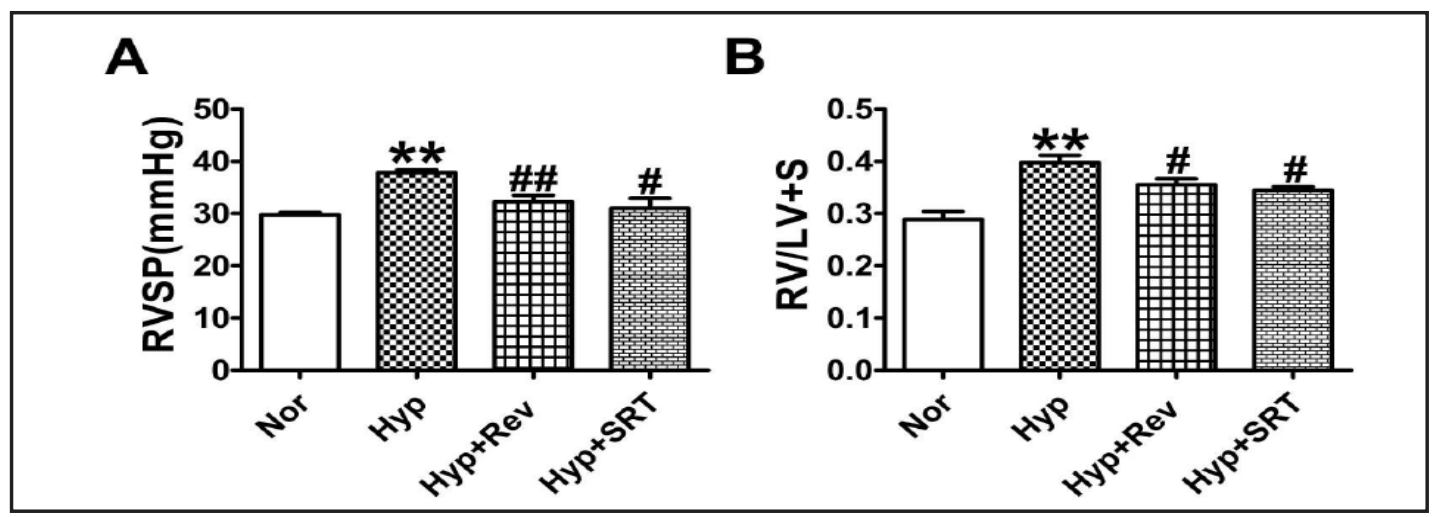

Fig. 6. Effects of Resveratrol (Rev) and SRT1720 on RVSP and Right ventricular hypertrophy. (A) The values for RVSP were measured in the different treatment groups. (B) Right ventricular hypertrophy (RVH) was also assessed. Data are expressed as the mean \pm SEM. ${ }^{* *} p<0.01$ vs. Nor group; \#\#p<0.01 or \#p<0.05 vs. Hyptreated group.

pretreatment with both Rev and SRT1720 also significantly attenuated right ventricular hypertrophy in vivo (Fig. 6B).

\section{Discussion}

Rev is a naturally occurring polyphenol present in many plant-based foods and is beneficial in various diseases. Currently, several studies have documented that Rev possesses various biological properties, including antioxidant, anti-inflammatory, and anti-proliferative properties, and promotes cell differentiation and apoptosis [27-30]. The data from a recent study showed that Rev reversed the remodelling of the pulmonary vasculature by restoring atrophic gene expression in MCT-induced PH [18]. In addition, Zhou et al. showed that Rev could elevate the activation of SIRT1 and p21 but decreased cyclin D1 expression in MCTinduced PAH [19].

The present study yielded several novel findings about the anti-PAH effects of Rev. In this study, the administration of Rev markedly reversed the remodelling of the pulmonary vasculature in rats exposed to hypoxia. Recent findings suggest that Rev is a non-specific activator of SIRT1 $[31,32]$. Whether SIRT1 is involved in hypoxia-induced PAH and in the anti-PAH effect mediated by Rev has not been fully disclosed until now. In this study, the rats received Rev for 21 days and the PASMCs were incubated with Rev for $24 \mathrm{~h}$ in a hypoxic environment. Immunoblotting was performed to show that the protein level of SIRT1 was predominantly decreased, but the non-specific activator of Rev could not reverse the level of SIRT1 protein expression as shown in Fig. 2. Therefore, we speculated that SIRT1 was involved in hypoxia-induced PAH, which could be associated with SIRT1 activation. PASMC proliferation and apoptosis play critical roles in $\mathrm{PAH}[3,33]$, indicating that anti-proliferative and apoptosis-promoting therapies are needed. Recently, multiple researchers have suggested that SIRT1 plays a crucial role in cell proliferation and apoptosis. For example, Igarashi et al. suggested that SIRT1 deacetylated S6K1, contributing to an increase in intestinal stem cell number. The upregulation of SIRT1 activity is closely related to certain health benefits [34]. In addition, the activation of the SIRT1 pathway contributes to the proliferation of vascular smooth muscle cells [35-37]. In contrast, multiple researchers have revealed that SIRT1 can induce cell apoptosis. Jin et al. demonstrated that both endogenous and exogenous SIRT1 were able to partially localize in the cytoplasm and that the cytoplasm-localized SIRT1 led to enhanced sensitivity to apoptosis [38]. Frazzi et al. found that Rev could increase Hodgkin lymphoma cells apoptosis, which was partially associated with SIRT1 activation [39]. Anti-proliferation and pro-apoptosis in PASMCs are considered beneficial in PAH, and multiple drugs have been discovered to prevent PAH by inducing PASMC apoptosis [40, 41]. 
Therefore, although Rev had no effect on the level of SIRT1 protein, its anti-PAH activity could be associated with enhancing SIRT1 activation, thus inhibiting PASMC proliferation and promoting their apoptosis. SRT1720 is considered to be a specific activator of SRT1720. Therefore, SRT1720 was chosen to further verify whether PAH was reversed by influencing the activation of SIRT1. To test this hypothesis, PASMCs were incubated with Rev and SRT1720. Consistent with our expectation, stimulation with different doses of Rev caused obvious decreases in cell proliferation (Fig. 1). To further verify the effect of Rev on PAH, different doses of SRT1720 were used. SRT1720 was found to decrease cell viability in a dose-dependent manner (Fig. 3). Funk et al. discovered that the activation of SIRT1 was predominantly enhanced by treatment with SRT1720 $(1 \mu \mathrm{m})$ in renal proximal tubule cells [42]. Yoshizaki et al. suggested that SRT1720 treatment $(1 \mu \mathrm{m})$ on adipocytes produced antiinflammatory effects and improved insulin sensitivity [43]. Consistent with our results, the dose of SRT1720 $(1 \mu \mathrm{m})$ also produced substantial outcomes. Therefore, a dose of SRT1720 $(1 \mu \mathrm{m})$ was used. Our results indicated that SRT1720 incubation substantially increased cell apoptosis (Fig. 4 and Fig. 5). Rao et al. reported that hypoxia could significantly enhance mitochondrial biogenesis and therefore increase the number of mitochondria [44]. Bonnet et al. indicated that hypoxia sensitivity in fawn hooded rats could engender an increased number of mitochondria in PASMCs [45]. In the current study, we provided evidence that Rev could lead to dysfunction in the mitochondria by inducing nuclear pyknosis and mitochondrial swelling. In the hypoxia treatment group, we found that the number of mitochondria was increased. The increased mitochondria could be associated with hypoxiainduced mitochondrial mitotic fission [46]. In addition, we further found that incubation with SRT1720 could alter mitochondrial permeability transition, thereby promoting PASMC apoptosis. The dosage of SRT1720 (25 mg/kg/day) was chosen according to several research studies. Zhou et al. suggested that a high dose of SRT1720 (50 mg/kg) could improve obesity induced by a high-fat diet in mice [47]. Minor et al. indicated that either a high dose of SRT1720 (100 mg/kg) or a low dose of SRT1720 (30 mg/kg) could improve survival and longevity in obese mice [48]. Therefore, the low dosage of SRT1720 (25 mg/kg/day) was used to assess whether SIRT1 activation reversed PAH in rats. Notably, we found that Rev and SRT1720 (25 mg/kg/day) treatment mitigated RVSP and reduced RVH.

The present study revealed the ability of Rev to mitigate PAH through involvement in the regulation of PASMC proliferation and apoptosis and through the activity of mitochondria by increasing SIRT1 activation. These findings may indicate a novel molecular mechanism underlying the anti-PAH effects of Rev. The results expanded our understanding of anti-PAH therapy, reconsolidating the view that Rev likely has critical functions and may be a novel therapeutic drug. However, the role of Rev in the anti-PAH pathway has not been deeply studied in our present work. Nevertheless, our results may lead to future studies that explore not only the implications of mitochondrial mitotic fission in anti-PAH mediated by Rev but also the beneficial effect of SIRT1 as a potential biological target for therapy in PAH. In addition, it should be noted that our study was performed in rats and that the findings cannot be extrapolated directly to humans. Therefore, appropriate caution is required in applying the results of this study to patients.

\section{Acknowledgments}

This work was supported, in part, by the National Natural Science Foundation of China (81300192, 81671746, 81503069, 81601150 and 81401457), the Special Financial Grant from the China Postdoctoral Science Foundation (2016T90313 and 2016T90316), the seed fund of Harbin Medical University (DQ2014-03, Daqing), the China Postdoctoral Science Foundation (2015M571449, 2015M581490 and 2014M561376), the Heilongjiang Health and Family Planning Commission (2014-385), the Postdoctoral Science Foundation of Heilongjiang Province (LBH-Z14143), and the Natural Science Foundation of Heilongjiang Province of China for Returnees (LC2015038). 


\section{Cellular Physiology Cell Physiol Biochem 2017;42:55-67 and Biochemistry Published onlıne: May 12, $2017 \quad$\begin{tabular}{l|l} 
DOI: 10.1159/000477115 2017 The Author(s). Published by S. Karger AG, Basel \\
www.karger.com/cpb
\end{tabular}}

Yu et al.: Resveratrol Prevents PAH through SIRT1 Activation

\section{Disclosure Statement}

The authors declare no competing financial interests.

\section{References}

1 Brittain EL, Talati M, Fessel JP, Zhu H, Penner N, Calcutt MW, West JD, Funke M, Lewis GD, Gerszten RE, Hamid R, Pugh ME, Austin ED, Newman JH, Hemnes AR: Fatty Acid Metabolic Defects and Right Ventricular Lipotoxicity in Human Pulmonary Arterial Hypertension. Circulation 2016;133:1936-1944.

-2 Gerges M, Gerges C, Pistritto AM, Lang MB, Trip P, Jakowitsch J, Binder T, Lang IM: Pulmonary Hypertension in Heart Failure. Epidemiology, Right Ventricular Function, and Survival. Am J Respir Crit Care Med 2015;192:1234-1246.

- 3 Deng L, Blanco FJ, Stevens H, Lu R, Caudrillier A, McBride M, McClure JD, Grant J, Thomas M, Frid M, Stenmark K, White K, Seto AG, Morrell NW, Bradshaw AC, MacLean MR, Baker AH: MicroRNA-143 Activation Regulates Smooth Muscle and Endothelial Cell Crosstalk in Pulmonary Arterial Hypertension. Circ Res 2015;117:870-883.

-4 Kim J, Kang Y, Kojima Y, Lighthouse JK, Hu X, Aldred MA, McLean DL, Park H, Comhair SA, Greif DM, Erzurum SC, Chun HJ: An endothelial apelin-FGF link mediated by miR-424 and miR-503 is disrupted in pulmonary arterial hypertension. Nat Med 2013;19:74-82.

-5 Barst RJ, Langleben D, Badesch D, Frost A, Lawrence EC, Shapiro S, Naeije R, Galie N: Treatment of pulmonary arterial hypertension with the selective endothelin-A receptor antagonist sitaxsentan. J Am Coll Cardiol 2006;47:2049-2056.

-6 Aubert JD, Juillerat-Jeanneret L: Endothelin-Receptor Antagonists beyond Pulmonary Arterial Hypertension: Cancer and Fibrosis. J Med Chem DOI:10.1021/acs.jmedchem.5b01781.

-7 Tapson VF, Jing ZC, Xu KF, Pan L, Feldman J, Kiely DG, Kotlyar E, McSwain CS, Laliberte K, Arneson C, Rubin LJ: Oral treprostinil for the treatment of pulmonary arterial hypertension in patients receiving background endothelin receptor antagonist and phosphodiesterase type 5 inhibitor therapy (the FREEDOM-C2 study): a randomized controlled trial. Chest 2013;144:952-958.

-8 Ogo T, Chowdhury HM, Yang J, Long L, Li X, Torres Cleuren YN, Morrell NW, Schermuly RT, Trembath RC, Nasim MT: Inhibition of overactive transforming growth factor-beta signaling by prostacyclin analogs in pulmonary arterial hypertension. Am J Respir Cell Mol Biol 2013;48:733-741.

-9 Blok IM, van Riel AC, Schuuring MJ, de Bruin-Bon RH, van Dijk AP, Hoendermis ES, Zwinderman AH, Mulder $\mathrm{BJ}$, Bouma BJ: The role of cystatin $\mathrm{C}$ as a biomarker for prognosis in pulmonary arterial hypertension due to congenital heart disease. Int J Cardiol 2016;209:242-247.

-10 Sardana M, Moll M, Farber HW: Novel investigational therapies for treating pulmonary arterial hypertension. Expert Opin Investig Drugs 2015;24:1571-1596.

-11 Antigny F, Hautefort A, Meloche J, Belacel-Ouari M, Manoury B, Rucker-Martin C, Pechoux C, Potus F, Nadeau V, Tremblay E, Ruffenach G, Bourgeois A, Dorfmuller P, Breuils-Bonnet S, Fadel E, Ranchoux B, Jourdon P, Girerd B, Montani D, Provencher S, Bonnet S, Simonneau G, Humbert M, Perros F: Potassium Channel Subfamily K Member 3 (KCNK3) Contributes to the Development of Pulmonary Arterial Hypertension. Circulation 2016;133:1371-1385.

12 Chen C, Wang Z, Wang L, Yang L, Ding M, Ding C, Sun Y, Lin Q, Huang X, Du X, Zhao X, Wang C: Puerarin induces mitochondria-dependent apoptosis in hypoxic human pulmonary arterial smooth muscle cells. PLoS One 2012;7:e34181.

13 Hansen T, Galougahi KK, Celermajer D, Rasko N, Tang 0, Bubb KJ, Figtree G: Oxidative and nitrosative signalling in pulmonary arterial hypertension - Implications for development of novel therapies. Pharmacol Ther DOI:10.1016/j.pharmthera.2016.05.005.

14 Wedgwood S, Lakshminrusimha S, Czech L, Schumacker PT, Steinhorn RH: Increased p22(phox)/Nox4 expression is involved in remodeling through hydrogen peroxide signaling in experimental persistent pulmonary hypertension of the newborn. Antioxid Redox Signal 2013;18:1765-1776. 


\section{Cellular Physiology Cell Physiol Biochem 2017;42:55-67 \begin{tabular}{l|l} 
DOI: 10.1159/000477115 & Ond Biochemistry 2017 The Author(s). Published by S. Karger AG, Basel \\
wubw.karger.com/cpb
\end{tabular}}

Yu et al.: Resveratrol Prevents PAH through SIRT1 Activation

15 Fike CD, Dikalova A, Slaughter JC, Kaplowitz MR, Zhang Y, Aschner JL: Reactive oxygen species-reducing strategies improve pulmonary arterial responses to nitric oxide in piglets with chronic hypoxia-induced pulmonary hypertension. Antioxid Redox Signal 2013;18:1727-1738.

16 Voelkel NF, Bogaard HJ, Al Husseini A, Farkas L, Gomez-Arroyo J, Natarajan R: Antioxidants for the treatment of patients with severe angioproliferative pulmonary hypertension? Antioxid Redox Signal 2013;18:1810-1817.

17 Fan Y, Chen M, Meng J, Yu L, Tu Y, Wan L, Fang K, Zhu W: Arsenic trioxide and resveratrol show synergistic anti-leukemia activity and neutralized cardiotoxicity. PLoS One 2014;9:e105890.

- 18 Paffett ML, Lucas SN, Campen MJ: Resveratrol reverses monocrotaline-induced pulmonary vascular and cardiac dysfunction: a potential role for atrogin-1 in smooth muscle. Vascul Pharmacol 2012;56:64-73.

-19 Zhou S, Li MT, Jia YY, Liu JJ, Wang Q Tian Z, Liu YT, Chen HZ, Liu DP, Zeng XF: Regulation of Cell Cycle Regulators by SIRT1 Contributes to Resveratrol-Mediated Prevention of Pulmonary Arterial Hypertension. Biomed Res Int 2015;2015:762349.

20 Csiszar A, Labinskyy N, Olson S, Pinto JT, Gupte S, Wu JM, Hu F, Ballabh P, Podlutsky A, Losonczy G, de Cabo R, Mathew R, Wolin MS, Ungvari Z: Resveratrol prevents monocrotaline-induced pulmonary hypertension in rats. Hypertension 2009;54:668-675.

21 Zhu D, Medhora M, Campbell WB, Spitzbarth N, Baker JE, Jacobs ER: Chronic hypoxia activates lung 15-lipoxygenase, which catalyzes production of 15-HETE and enhances constriction in neonatal rabbit pulmonary arteries. Circ Res 2003;92:992-1000.

22 Yao L, Nie X, Shi S, Song S, Hao X, Li S, Zhu D: Reciprocal regulation of HIF-1alpha and 15-LO/15-HETE promotes anti-apoptosis process in pulmonary artery smooth muscle cells during hypoxia. Prostaglandins Other Lipid Mediat 2012;99:96-106.

23 Yu X, Li T, Liu X, Yu H, Hao Z, Chen Y, Zhang C, Liu Y, Li Q Mao M, Zhu D: Modulation of Pulmonary Vascular Remodeling in Hypoxia: Role of 15-LOX-2/15-HETE-MAPKs Pathway. Cell Physiol Biochem 2015;35:20792097.

-24 Abe K, Shimokawa H, Morikawa K, Uwatoku T, Oi K, Matsumoto Y, Hattori T, Nakashima Y, Kaibuchi K, Sueishi K, Takeshit A: Long-term treatment with a Rho-kinase inhibitor improves monocrotaline-induced fatal pulmonary hypertension in rats. Circ Res 2004;94:385-393.

25 Fulton RM, Hutchinson EC, Jones AM: Ventricular weight in cardiac hypertrophy. Br Heart J 1952;14:413420.

26 Tait SW, Green DR: Mitochondria and cell death: outer membrane permeabilization and beyond. Nat Rev Mol Cell Biol 2010;11:621-632.

27 Deng W, Cha J, Yuan J, Haraguchi H, Bartos A, Leishman E, Viollet B, Bradshaw HB, Hirota Y, Dey SK: p53 coordinates decidual sestrin 2/AMPK/mTORC1 signaling to govern parturition timing. J Clin Invest DOI:10.1172/JCI87715.

28 Fuggetta MP, Bordignon V, Cottarelli A, Macchi B, Frezza C, Cordiali-Fei P, Ensoli F, Ciafre S, Marino-Merlo F, Mastino A, Ravagnan G: Downregulation of proinflammatory cytokines in HTLV-1-infected T cells by Resveratrol. J Exp Clin Cancer Res 2016;35:118.

29 Sinha D, Sarkar N, Biswas J, Bishayee A: Resveratrol for breast cancer prevention and therapy: Preclinical evidence and molecular mechanisms. Semin Cancer Biol DOI:10.1016/j.semcancer.2015.11.001.

- 30 Deus CM, Serafim TL, Magalhaes-Novais S, Vilaca A, Moreira AC, Sardao VA, Cardoso SM, Oliveira PJ: Sirtuin 1-dependent resveratrol cytotoxicity and pro-differentiation activity on breast cancer cells. Arch Toxicol DOI:10.1007/s00204-016-1784-X.

-31 Borra MT, Smith BC, Denu JM: Mechanism of human SIRT1 activation by resveratrol. J Biol Chem 2005;280:17187-17195.

- 32 Arunachalam G, Yao H, Sundar IK, Caito S, Rahman I: SIRT1 regulates oxidant- and cigarette smoke-induced eNOS acetylation in endothelial cells: Role of resveratrol. Biochem Biophys Res Commun 2010;393:66-72.

33 Omura J, Satoh K, Kikuchi N, Satoh T, Kurosawa R, Nogi M, Otsuki T, Kozu K, Numano K, Suzuki K, Sunamura S, Tatebe S, Aoki T, Sugimura K, Miyata S, Hoshikawa Y, Okada Y, Shimokawa H: Protective Roles of Endothelial AMP-Activated Protein Kinase Against Hypoxia-Induced Pulmonary Hypertension in Mice. Circ Res 2016;119:197-209.

-34 Igarashi M, Guarente L: mTORC1 and SIRT1 Cooperate to Foster Expansion of Gut Adult Stem Cells during Calorie Restriction. Cell 2016;166:436-450. 


\section{Cellular Physiology Cell Physiol Biochem 2017;42:55-67 \begin{tabular}{l|l} 
DOI: 10.1159/000477115 & $\begin{array}{l}\text { O 2017 The Author(s). Published by S. Karger AG, Basel } \\
\text { www.karger.com/cpb }\end{array}$
\end{tabular}}

Yu et al.: Resveratrol Prevents PAH through SIRT1 Activation

35 Ma Y, Gong X, Mo Y, Wu S: Polydatin inhibits the oxidative stress-induced proliferation of vascular smooth muscle cells by activating the eNOS/SIRT1 pathway. Int J Mol Med 2016;37:1652-1660.

-36 Shan W, Gao L, Zeng W, Hu Y, Wang G, Li M, Zhou J, Ma X, Tian X, Yao J: Activation of the SIRT1/p66shc antiapoptosis pathway via carnosic acid-induced inhibition of miR-34a protects rats against nonalcoholic fatty liver disease. Cell Death Dis 2015;6:e1833.

- 37 Yamakuchi M, Ferlito M, Lowenstein CJ: miR-34a repression of SIRT1 regulates apoptosis. Proc Natl Acad Sci U S A 2008;105:13421-13426.

- 38 Jin Q, Yan T, Ge X, Sun C, Shi X, Zhai Q: Cytoplasm-localized SIRT1 enhances apoptosis. J Cell Physiol 2007;213:88-97.

39 Frazzi R, Valli R, Tamagnini I, Casali B, Latruffe N, Merli F: Resveratrol-mediated apoptosis of hodgkin lymphoma cells involves SIRT1 inhibition and FOXO3a hyperacetylation. Int J Cancer 2013;132:1013-1021.

40 McMurtry MS, Bonnet S, Wu X, Dyck JR, Haromy A, Hashimoto K, Michelakis ED: Dichloroacetate prevents and reverses pulmonary hypertension by inducing pulmonary artery smooth muscle cell apoptosis. Circ Res 2004;95:830-840.

41 Zhang Q, Fan K, Wang P, Yu J, Liu R, Qi H, Sun H, Cao Y: Carvacrol induces the apoptosis of pulmonary artery smooth muscle cells under hypoxia. Eur J Pharmacol 2016;770:134-146.

-42 Funk JA, Odejinmi S, Schnellmann RG: SRT1720 induces mitochondrial biogenesis and rescues mitochondrial function after oxidant injury in renal proximal tubule cells. J Pharmacol Exp Ther 2010;333:593-601.

43 Yoshizaki T, Milne JC, Imamura T, Schenk S, Sonoda N, Babendure JL, Lu JC, Smith JJ, Jirousek MR, Olefsky JM: SIRT1 exerts anti-inflammatory effects and improves insulin sensitivity in adipocytes. Mol Cell Biol 2009;29:1363-1374.

-44 Rao J, Li J, Liu Y, Lu P, Sun X, Sugumaran PK, Zhu D: The key role of PGC-1alpha in mitochondrial biogenesis and the proliferation of pulmonary artery vascular smooth muscle cells at an early stage of hypoxic exposure. Mol Cell Biochem 2012;367:9-18.

-45 Bonnet S, Michelakis ED, Porter CJ, Andrade-Navarro MA, Thebaud B, Haromy A, Harry G, Moudgil R, McMurtry MS, Weir EK, Archer SL: An abnormal mitochondrial-hypoxia inducible factor-1alpha-Kv channel pathway disrupts oxygen sensing and triggers pulmonary arterial hypertension in fawn hooded rats: similarities to human pulmonary arterial hypertension. Circulation 2006;113:2630-2641.

-46 Marsboom G, Toth PT, Ryan JJ, Hong Z, Wu X, Fang YH, Thenappan T, Piao L, Zhang HJ, Pogoriler J, Chen Y, Morrow E, Weir EK, Rehman J, Archer SL: Dynamin-related protein 1-mediated mitochondrial mitotic fission permits hyperproliferation of vascular smooth muscle cells and offers a novel therapeutic target in pulmonary hypertension. Circ Res 2012;110:1484-1497.

47 Zhou XL, Xu JJ, Ni YH, Chen XC, Zhang HX, Zhang XM, Liu WJ, Luo LL, Fu YC: SIRT1 activator (SRT1720) improves the follicle reserve and prolongs the ovarian lifespan of diet-induced obesity in female mice via activating SIRT1 and suppressing mTOR signaling. J Ovarian Res 2014;7:97.

- 48 Minor RK, Baur JA, Gomes AP, Ward TM, Csiszar A, Mercken EM, Abdelmohsen K, Shin YK, Canto C, Scheibye-Knudsen M, Krawczyk M, Irusta PM, Martin-Montalvo A, Hubbard BP, Zhang Y, Lehrmann E, White AA, Price NL, Swindell WR, Pearson KJ, Becker KG, Bohr VA, Gorospe M, Egan JM, Talan MI, Auwerx J, Westphal CH, Ellis JL, Ungvari Z, Vlasuk GP, Elliott PJ, Sinclair DA, de Cabo R: SRT1720 improves survival and healthspan of obese mice. Sci Rep 2011;1:70. 